\title{
State estimation of the three-tank system using a multiple model
}

\author{
Anca Maria Nagy, Benoît Marx, Gilles Mourot, Georges Schutz, José Ragot
}

\begin{abstract}
This paper addresses the exact transformation of nonlinear systems into a multiple model form with unmeasurable premise variables. The multiple model structure serves to treat the observability and the state estimation problem of nonlinear systems. Using a method with no information loss, a nonlinear system is transformed into a multiple model, depending on the choice of premise variables. It is a key point, since it allows to choose, between different multiple model forms, the one that has suitable structure and properties, in order to design an observer. The convergence conditions of the state estimation error are expressed in LMI formulation using the Lyapunov method. These proposals are investigated and applied to the three-tank system.
\end{abstract}

\section{INTRODUCTION}

The fault detection and diagnosis (FDD) methods for linear systems had already reached a certain development and maturity. Nevertheless, nonlinear models are often needed to represent real system behaviors. As a consequence, there is a need to extend FDD methods to nonlinear systems, even if it is a difficult point. It is the reason why the concept of multiple models has received attention in the last two decades. The multiple model structure gives the possibility to reduce the complexity of nonlinear systems, by constructing linear submodels aggregated using weighting functions [16], [14]. The interests to use this structure is already well known, the main advantage is the possibility to extend many tools and results of the linear theory to nonlinear systems, such as the observer synthesis.

In the linear framework, the fault detection can be realized using observer based methods. There already exists several works on observer design for multiple model systems. A multi-observer based on Luenberger observers was proposed and applied for diagnosis in [12]. In [2], sliding mode observers for linear systems, were transposed to the multiple model case, and in [2], [3] unknown input multi-observers were implemented. Moreover, one of the main interests to use this type of observers is its robustness with regard to modeling uncertainties.

However, these papers assume that the weighting functions depend on measurable premise variables, which is not always the case. In the literature, few works are devoted to the case of unmeasurable decision variables [5], [7], [10]. As discussed in [7], multiple model with unmeasurable decision

Anca Maria Nagy, Benoît Marx, Gilles Mourot, José Ragot are with Centre de Recherche en Automatique de Nancy, Nancy Université, 2, avenue de la Forêt de Haye, 54516 Vandoeuvre-lesNancy Cedex France anca-maria.nagy, gilles.mourot, benoit.marx, jose.ragoteensem.inpl-nancy.fr

Georges Schutz is with CRP "Henri Tudor", LTI Unité Business and Systems Analytics, 29, Avenue John F. Kennedy, L-1855 Luxembourg Kirchberg georges.schutzltudor. Iu variables is more adequate to fault diagnosis since a single model can be used, both for sensor and actuator fault diagnosis. [7] proposes a method which reduces the conservatism of the precedent works by reducing the number of LMIs to be solved and relaxing the conditions under which the method is applicable. In addition, the number of LMIs does not depend on the number of submodels of the multiple model.

Different techniques to obtain a multiple model exist in the literature. Firstly, model identification techniques or parameter estimation using experimental data [1] can be considered. Secondly, the techniques based on existent mathematical nonlinear models, where a linearization around one (or several) operating point(s) as well as a dynamic linearization near an arbitrary trajectory are proposed[8]. The main drawbacks of this technique are: the loss of information, the choice of the premise variables expressing different nonlinearities of the system is not systematically realized, the choice of different operating points still remains very delicate. The method used here avoids this particular loss and gives an equivalent multiple model form of the initial nonlinear system [9]. In addition to that, the method offers several multiple models with different structures, depending on the choice of premise variables. So, it is possible to choose from different multiple model structures the one that ensures the convergence of the multi-observer, with respect to given conditions. The method is here improved and adapted in order to facilitate the choice of the suitable MM structure, by introducing a pondered sum of the state variables when constructing the MM form. The convergence conditions of the state estimation error are expressed in LMI formulation using the Lyapunov method. The method used here for the observer design [7] requires the observability of the multiple model. It is the reason why an observable multiple model structure is sought.

These proposals are investigated and applied in this paper to the three-tank system. This system was largely studied in the literature [11], [13], [17] and is considered as a benchmark, in different frameworks. The controller switching approach used for fault tolerant control in [13], implies a period of time between two switchings (dwell time). In [11] a sliding mode observer for the three-tank system is proposed, where a particular region of operation is considered. The method proposed in this paper, avoids the need to separate different operating modes and give a multiple model which encompasses all possible modes, without any transition management. In [17] a fault diagnosis and accommodation method is applied to the three-tank system, that is linearized around an operating point using Taylor expansion. It is well known that the choice of the different operating points still 
remains a very delicate subject. The method proposed in this paper consists in rewriting the nonlinear models. As a consequence no operating points have to be chosen.

In order to ensure the possibility to design an observer, a preliminary study of observability for nonlinear systems has to be realized. The uniform observability notion is closely related to observer design. There are different approaches to verify this condition. In [6] a systematic observability analysis for the three-tank system is realized. As a consequence, the observer design for the three-tank system is possible.

The paper is organized as follows. Section II starts with the presentation of the three-tank system in II-A. In section II-B the main steps of the method used to obtain an equivalent multiple model form of a nonlinear system (here, the threetank system) are given. This method proposes a choice criterion of multiple models with suitable structure and properties, in order to design a multi-observer (section IIC). The design of the proposed observer based on multiple model form is described in section II-D. In section III some conclusions and work perspectives are given.

\section{THE THREE-TANK SYSTEM}

\section{A. Process description}

The three-tank system is a MIMO system with two inputs and two outputs, presented in Fig. 1. The system consists in three cylindrical tanks of the same section $S$. They are linked to each other through connecting cylindrical pipes having the section $S_{n}$. The nominal outlet is located at tank 3. The incoming flows $Q_{1}$ and $Q_{2}$ are considered as the inputs of the system. The control objective is to regulate the water levels in tanks 1 and 2 by adjusting $Q_{1}$ and $Q_{2} . \alpha_{i}(i=1, \ldots, 3)$ are the outflow coefficients, $g=9.82\left[\mathrm{~m} / \mathrm{s}^{2}\right]$ the gravitational acceleration. The objectives here are to analyze observability of the system based on single or double measurements and to design observers for estimating all the tank levels. The

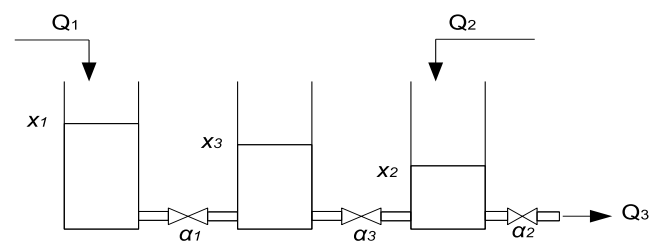

Fig. 1. The three-tank system

dynamic mathematical model is based on Bernoulli's law for liquids and on mass conservation law:

$$
\begin{aligned}
\dot{x}_{1}(t)= & \frac{Q_{1}(t)}{S}-\frac{\alpha_{1} S_{n}}{S} \operatorname{sgn}\left[x_{1}(t)-x_{3}(t)\right] \sqrt{2 g\left|x_{1}(t)-x_{3}(t)\right|} \\
\dot{x}_{2}(t)= & \frac{Q_{2}(t)}{S}+\frac{\alpha_{3} S_{n}}{S} \operatorname{sgn}\left[x_{3}(t)-x_{2}(t)\right] . \\
& \sqrt{2 g\left|x_{3}(t)-x_{2}(t)\right|}-\frac{Q_{3}(t)}{S} \\
\dot{x}_{3}(t)= & \frac{\alpha_{1} S_{n}}{S} \operatorname{sgn}\left[x_{1}(t)-x_{3}(t)\right] \sqrt{2 g\left|x_{1}(t)-x_{3}(t)\right|} \\
& -\frac{\alpha_{3} S_{n}}{S} \operatorname{sgn}\left[x_{3}(t)-x_{2}(t)\right] \sqrt{2 g\left|x_{3}(t)-x_{2}(t)\right|}
\end{aligned}
$$

where $O_{3}(t)=\alpha_{2} S_{n} \sqrt{2 g x_{2}(t)}$ and $\operatorname{son}(\cdot)$ denotes the (1) function. For the sake of the simplicity, let us note:

$$
W_{i, k}=\alpha_{i} \frac{S_{n}}{S} \operatorname{sgn}\left[x_{i}(t)-x_{k}(t)\right], i \neq k
$$

Define

$$
x=\left[\begin{array}{l}
x_{1} \\
x_{2} \\
x_{3}
\end{array}\right], \quad u=\left[\begin{array}{l}
Q_{1} \\
Q_{2}
\end{array}\right]
$$

Let us consider $H$ the maximal liquid level in the tanks and suppose that the tanks are never simultaneously empty. This assumption can be made because the levels $x_{1}, x_{2}$ and $x_{3}$ are controllable using the flows $Q_{1}$ and $Q_{2}$. In this case, the three-tank levels $x_{1}, x_{2}$ and $x_{3}$ are bounded: $x \in \mathbb{R}_{+}^{3}$.

\section{B. The multiple model representation}

Let us consider the multiple model representation of a nonlinear system:

$$
\left\{\begin{aligned}
\dot{x}(t) & =\sum_{i=1}^{r} \mu_{i}(\xi(t))\left[A_{i} x(t)+B_{i} u(t)\right] \\
y(t) & =C x(t)
\end{aligned}\right.
$$

$A_{i}, B_{i}$ and $C$ are real known matrices with appropriate dimensions. The weighting functions $\mu_{i}(\cdot)$ are generally nonlinear in $\xi(t)$ and satisfy the convexity property:

$$
\begin{aligned}
& \sum_{i=1}^{r} \mu_{i}(\xi(t))=1, \quad \forall t \\
& 0 \leq \mu_{i}(\xi(t)) \leq 1, i \in\{1, \ldots, r\}, \quad \forall t
\end{aligned}
$$

The premise variable $\xi(t)$ can depend on measurable variables, as the input $u(t)$ or the output $y(t)$ of the system, and/or on unmeasurable variables like the state $x(t)$.

In order to obtain a multiple model structure from a given nonlinear system different methods exists. The majority of them are linearization based techniques, which come with an information loss of the initial nonlinear system. The method used here avoids this particular loss and gives an equivalent multiple model form of the initial nonlinear system. Only a brief description of the important points of this method will be given as follows, the technical details can be found in [9]. Considering a nonlinear system with bounded nonlinearities, it can be written in a quasi linear-parameter-varying form:

$$
\left\{\begin{array}{l}
\dot{x}(t)=A(\kappa(t)) x(t)+B(\kappa(t)) u(t) \\
y(t)=C x(t)
\end{array}\right.
$$

where $\kappa(t)$ can represent a subset of the state variables. To each quasi-LPV form corresponds a particular set of premise variables and a particular multiple model structure. As a consequence, it is possible to choose between different multiple models the one with convenient properties. This represents a degree of freedom that will be used to make easier the construction of an observer.

The observer stability conditions are given in terms of linear matrix inequalities (LMI). Different techniques are proposed to reduce the number of LMI conditions, which leads to less computational requirement [15] and ensures the solution existence [5]. Due to the convexity of the interpolation made between the submodels, the number of LMI to be satisfied is linear or polynomial in the number of submodels [14]. Therefore, it is tried to find a multiple model composed by a minimal number of submodels. This number is related to the number of premise variables, so that a quasi-LPV 
form which has a minimal set of premise variables will be preferred. Besides, the complexity of the LMI conditions increases with the number of state variables involved in the premise variables. In order to ensure the solution existence, the dimension of the state vector involved in the premise variables should be made as small as possible [4].

Taking into account the previous remarks, the choice of the quasi-LPV form leading to the simplest observable multiple model will be realized, using the following rules:

1) Eliminate all quasi-LPV forms for which the matrices A and/or B have null rows and/or columns.

2) Identify the decompositions containing common premise variables for different state equations, reducing in this way the number of premise variables.

3) Chose the quasi-LPV form for which the premise variables depend on a minimal number of state variables.

The premise variables associated to the quasi-LPV form will be partitioned into two parts, using the convex polytopic transformation. The two partitions of each bounded nonlinearity will contribute to the construction of the submodels and to the corresponding weighting functions. The multiple model will thus be a convex combination of linear submodels, the nonlinearity being transferred into weighting functions related to each submodel.

In the following, the previous essential points of these method will be considered in order to transform the threetank system into a multiple model form. Nevertheless, a new technique will be implemented concerning the quasi-LPV forms. These forms will be highlighted through a weighted sum of the state variables.

The three-tank system is rewritten in an equivalent way, using a weighted sum of the state variables, via the real scalars $\lambda_{i}$ and $\gamma_{i}(i=1, \ldots, n)$. The choice of real scalars $\lambda_{i}$ and $\gamma_{i}$ gives the possibility to obtain different multiple model structures. In fact, the use of these scalars allows to highlight the existence of different quasi-LPV forms of the system. Taking into account the notations (2) the three-tank system (1) is then rewritten:

$$
\begin{aligned}
\dot{x}_{1}(t)= & \frac{1}{S} Q_{1}(t)-\frac{W_{1,3}(t)}{\sum_{i=1}^{3} \lambda_{i} x_{i}(t)} \sqrt{2 g\left|x_{1}(t)-x_{3}(t)\right|} \sum_{i=1}^{3} \lambda_{i} x_{i}(t) \\
\dot{x}_{2}(t)= & \frac{1}{S} Q_{2}(t)-\frac{\alpha_{2} S_{n}}{S} \sqrt{2 g x_{2}(t)} \\
& +\frac{W_{3,2}(t)}{\sum_{i=1}^{3} \gamma_{i} x_{i}(t)} \sqrt{2 g\left|x_{3}(t)-x_{2}(t)\right|} \sum_{i=1}^{3} \gamma_{i} x_{i}(t) \\
\dot{x}_{3}(t)= & \frac{W_{1,3}(t)}{\sum_{i=1}^{3} \lambda_{i} x_{i}(t)} \sqrt{2 g\left|x_{1}(t)-x_{3}(t)\right|} \sum_{i=1}^{3} \lambda_{i} x_{i}(t) \\
& -\frac{W_{3,2}(t)}{\sum_{i=1}^{3} \gamma_{i} x_{i}(t)} \sqrt{2 g\left|x_{3}(t)-x_{2}(t)\right|} \sum_{i=1}^{3} \gamma_{i} x_{i}(t)
\end{aligned}
$$

for non null $\lambda_{i}$ and $\gamma_{i}$, and since $x(t)$ is non null, then $\sum_{i=1}^{3} \lambda_{i} x_{i}(t)$ and $\sum_{i=1}^{3} \gamma_{i} x_{i}(t)$ are not null.

Let us define the premise variables:

$$
\begin{aligned}
& \mathbf{z}_{\mathbf{1}}(x)=\frac{W_{1,3}(t)}{\sum_{i=1}^{3} \lambda_{i} x_{i}} \sqrt{2 g\left|x_{1}-x_{3}\right|} \\
& \mathbf{z}_{2}(x)=\frac{\alpha_{2} S_{n}}{S} \sqrt{\frac{2 g}{x_{2}}} \\
& \mathbf{z}_{3}(x)=\frac{W_{3,2}(t)}{\sum_{i=1}^{3} \gamma_{i} x_{i}} \sqrt{2 g\left|x_{3}-x_{2}\right|}
\end{aligned}
$$

where $x_{i}=x_{i}(t), i=1, \ldots, 3$.

The system (7) takes the following quasi-LPV form:

$$
\dot{x}(t)=A\left(z_{1}, z_{2}, z_{3}\right) x(t)+B u(t)
$$

where

$$
\begin{gathered}
A\left(z_{1}, z_{2}, z_{3}\right)=\left[\begin{array}{ccc}
-\lambda_{1} \mathbf{z}_{1} & -\lambda_{2} \mathbf{z}_{1} & -\lambda_{3} \mathbf{z}_{\mathbf{1}} \\
\gamma_{1} \mathbf{z}_{\mathbf{3}} & \gamma_{2} \mathbf{z}_{\mathbf{3}}-\mathbf{z}_{\mathbf{2}} & \gamma_{3} \mathbf{z}_{\mathbf{3}} \\
\lambda_{1} \mathbf{z}_{\mathbf{1}}-\gamma_{1} \mathbf{z}_{\mathbf{3}} & \lambda_{2} \mathbf{z}_{\mathbf{1}}-\gamma_{2} \mathbf{z}_{\mathbf{3}} & \lambda_{3} \mathbf{z}_{\mathbf{1}}-\gamma_{3} \mathbf{z}_{\mathbf{3}}
\end{array}\right] \\
B=\left[\begin{array}{cc}
1 / S & 0 \\
0 & 1 / S \\
0 & 0
\end{array}\right]
\end{gathered}
$$

Using the convex polytopic transformation [14], the three premise variables $z_{j}$ are divided into two parts:

$$
\begin{aligned}
\mathbf{z}_{j}(x) & =\frac{\mathbf{z}_{j}(x)-z_{j, 2}}{z_{j, 1}-z_{j, 2}} z_{j, 1}+\frac{z_{j, 1}-\mathbf{z}_{j}(x)}{z_{j, 1}-z_{j, 2}} z_{j, 2} \\
& =F_{j, 1}\left(\mathbf{z}_{j}(x)\right) z_{j, 1}+F_{j, 2}\left(\mathbf{z}_{j}(x)\right) z_{j, 2}
\end{aligned}
$$

where the scalars $z_{j, 1}$ and $z_{j, 2}$ are:

$$
\begin{aligned}
& z_{j, 1}=\max _{x}\left\{\mathbf{z}_{j}(x)\right\} \\
& z_{j, 2}=\min _{x}\left\{\mathbf{z}_{j}(x)\right\}
\end{aligned} \quad \forall j=1, \ldots, 3
$$

The multiple model is composed of $8=2^{3}$ submodels. The corresponding weighting functions are calculated as the products of the partition functions $F_{j, \sigma_{i}^{j}}(\cdot)$ from (12) (where $\sigma_{i}^{j}$ can take values 1 or 2 ) in order to obtain all 8 possible combinations between the functions:

$$
\mu_{i}(x)=F_{1, \sigma_{i}^{1}}\left(\mathbf{z}_{\mathbf{1}}(x)\right) F_{2, \sigma_{i}^{2}}\left(\mathbf{z}_{\mathbf{2}}(x)\right) F_{3, \sigma_{i}^{3}}\left(\mathbf{z}_{\mathbf{3}}(x)\right)
$$

with $\left(\sigma_{i}^{1}, \sigma_{i}^{2}, \sigma_{i}^{3}\right)=\sigma_{i}$ corresponding to the submodel $i$ is given in the table I and indicates which of the partition functions $F_{j, \sigma_{i}^{j}}(\cdot)$ will be used in the computation of the weights $\mu_{i}(x)$. Similarly, the matrices $A_{i}(i=1, \ldots, 8)$ are obtained using the scalars $z_{j, \sigma_{i}^{j}}(j=1, \ldots, 3)$ expressed in (13) in order to obtain the 8 possible combinations:

$$
A_{i}=A\left(z_{1, \sigma_{i}^{1}}, z_{2, \sigma_{i}^{2}}, z_{3, \sigma_{i}^{3}}\right) \quad i=1, \ldots, 8
$$

where the matrix $A\left(z_{1}, z_{2}, z_{3}\right)$ is given in (10). Considering (11), all the matrices $B_{i}$ are identical and thus the matrices $B_{i}=B(i=1, \ldots, 8)$. 
TABLE I

MULTIPLE MODEL CONSTRUCTION USING THE PARTITIONS OF THE THREE PREMISE VARIABLES

\begin{tabular}{|c|c|c|c|c|c|c|c|}
\hline \multirow{3}{*}{$\begin{array}{c}\text { Model } \\
i\end{array}$} & \multicolumn{6}{|c|}{ Partitions } & \multirow{3}{*}{$\sigma_{i}$} \\
\hline & \multicolumn{2}{|c|}{$z_{1}$} & \multicolumn{2}{|c|}{$z_{2}$} & \multicolumn{2}{|c|}{$z_{3}$} & \\
\hline & $F_{1, \mathbf{1}}$ & $F_{1,2}$ & $F_{2, \mathbf{1}}$ & $F_{2,2}$ & $F_{3,1}$ & $F_{3,2}$ & \\
\hline 1 & 1 & 0 & 1 & 0 & 1 & 0 & $(1,1,1)$ \\
\hline 2 & 1 & 0 & 1 & 0 & 0 & 1 & $(1,1,2)$ \\
\hline 3 & 1 & 0 & 0 & 1 & 1 & 0 & $(1,2,1)$ \\
\hline 4 & 1 & 0 & 0 & 1 & 0 & 1 & $(1,2,2)$ \\
\hline 5 & 0 & 1 & 1 & 0 & 1 & 0 & $(2,1,1)$ \\
\hline 6 & 0 & 1 & 1 & 0 & 0 & 1 & $(2,1,2)$ \\
\hline 7 & 0 & 1 & 0 & 1 & 1 & 0 & $(2,2,1)$ \\
\hline 8 & 0 & 1 & 0 & 1 & 0 & 1 & $(2,2,2)$ \\
\hline
\end{tabular}

Then, the three-tank model (1) is transformed into the multiple model:

$$
\begin{aligned}
\dot{x}(t) & =\sum_{i=1}^{8} \mu_{i}(x(t))\left[A_{i} x(t)+B u(t)\right] \\
y(t) & =C x(t)
\end{aligned}
$$

It has to be remarked that in conformity with (8) and (10) the weighting functions $\mu_{i}(x)$ and the matrices $A_{i}$ depend on the real scalars $\lambda_{i}$ and $\gamma_{i}(i=1,2,3)$. So, for different choices of these scalars, different structures of the multiple model will be obtained. All these multiple model structures (excluding the degenerated cases $\lambda_{1}=\lambda_{2}=\lambda_{3}=0$ and/or $\gamma_{1}=\gamma_{2}=\gamma_{3}=0$ ) are analytically equivalent with the initial nonlinear form of the three-tank model (1).

In the next section will be discussed the conditions to be respected when choosing the real scalars $\lambda_{i}$ and $\gamma_{i}(i=$ $1,2,3$ ) (and so the multiple model form) in order to design an observer for the three-tank system using the observer proposed in [7].

\section{Observability criterion choice of the multiple model}

In order to design an observer for a multiple model, the observability of the system is required to solve the LMI, given in [7], and obtain the gains of the observer.

It is well known that the observability of each submodel is a requirement for the observability of the global multiple model. The next geometric conditions are used for the multiple model (16):

$$
\operatorname{rank}\left(\mathscr{O}_{i}\right)=\operatorname{rank}\left[\begin{array}{c}
C \\
C A_{i} \\
\vdots \\
C A_{i}^{n-1}
\end{array}\right]=n, \quad \forall i=1, \ldots, 8
$$

In conformity with $(15)$ the matrices $A_{i}(i=1, \ldots, 8)$ have the same structure as that of the matrix $A$ given in (10), with the difference that $z_{1}, z_{2}$ and $z_{3}$ are replaced with constants representing the maximum or the minimum of the three premise variables $\left(z_{1, \sigma_{i}^{1}}, z_{2, \sigma_{i}^{2}}\right.$ or $\left.z_{3, \sigma_{i}^{3}}\right)$. Taking into account that the degenerate cases $\left(x_{1}=x_{3}, x_{2}=x_{3}\right.$ and $x_{1}=x_{2}=x_{3}=0$ ) are excluded, the maximum and the minimum of the premise variables are always non null. As a consequence, the eight conditions (17) are verified if the same condition is verified for the matrix $A$.

Firstly, for the sake of simplicity, only one measure will be considered, for example $x_{1}$, which defines the matrix $C=\left[\begin{array}{lll}1 & 0 & 0\end{array}\right]$.

It has to be remarked that the observer design based on a single measure may be interesting for fault diagnosis studies. The observability matrix $\mathscr{O}^{1}$ for the matrix $A$ has the following form:

$$
\mathscr{O}^{1}=\left[\begin{array}{c}
C \\
C A \\
C A^{2}
\end{array}\right]=\left[\begin{array}{ccc}
1 & 0 & 0 \\
-\lambda_{1} z_{1} & -\lambda_{2} z_{1} & -\lambda_{3} z_{1} \\
(*)_{1} & (*)_{2} & (*)_{3}
\end{array}\right]
$$

where $*$ represent non null terms:

$$
\begin{aligned}
& (*)_{1}=\lambda_{1}^{2} z_{1}^{2}-\lambda_{3} z_{1}\left(\lambda_{1} z_{1}-\gamma_{1} z_{3}\right)-\lambda_{2} z_{1} \gamma_{1} z_{3} \\
& (*)_{2}=\lambda_{1} z_{1}^{2} \lambda_{2}-\lambda_{2} z_{1}\left(\gamma_{2} z_{3}-z_{2}\right)-\lambda_{3} z_{1}\left(\lambda_{2} z_{1}-\gamma_{2} z_{3}\right) \\
& (*)_{3}=\lambda_{1} \lambda_{3} z_{1}^{2}-\lambda_{3} z_{1}\left(\lambda_{3} z_{1}-\gamma_{3} z_{3}\right)-\lambda_{2} z_{1} \gamma_{3} z_{3}
\end{aligned}
$$

The determinant of the observability matrix $\mathscr{O}^{1}$ is in this case $\operatorname{det}\left(\mathscr{O}^{1}\right)=\lambda_{2} \gamma_{3} z_{1}^{2} z_{3}\left(\lambda_{2}-\lambda_{3}\right)-\lambda_{3} \lambda_{2} z_{1}^{2}\left(\gamma_{2} z_{3}+z_{2}\right)+\lambda_{3}^{2} \gamma_{2} z_{1}^{2} z_{3}$. It can be deduced that the rank of the observability matrix $\mathscr{O}^{1}$ is equal to $n=3$ if and only if $\lambda_{3} \neq 0$ and $\lambda_{2} \neq 0$. For $\lambda_{3}, \lambda_{2} \neq 0$ each submodel is observable, which is necessary for observer design.

Secondly, it can be considered the case of two measured outputs. If the classical case of the three-tank system is considered, where $x_{1}$ and $x_{2}$ are the measured outputs, the matrix $C$ has the form:

$$
C=\left[\begin{array}{lll}
1 & 0 & 0 \\
0 & 1 & 0
\end{array}\right]
$$

The observability matrix $\mathscr{O}^{1,2}(i=1, \ldots, 8)$ has the following form:

$$
\mathscr{O}^{1,2}=\left[\begin{array}{ccc}
1 & 0 & 0 \\
0 & 1 & 0 \\
-\lambda_{1} z_{1} & -\lambda_{2} z_{2} & -\lambda_{3} z_{1} \\
\gamma_{1} z_{3} & \gamma_{2} z_{3}-z_{2} & \gamma_{3} z_{3} \\
\lambda_{1} z_{1}^{2}\left(\lambda_{1}-\lambda_{3}\right)+\gamma_{1} \lambda_{3} z_{1} z_{3} & (*)_{1} & (*)_{2} \\
(*)_{3} & (*)_{4} & (*)_{5}
\end{array}\right]
$$

where $*$ represent the following non null terms:

$$
\begin{aligned}
& (*)_{1}=z_{1}\left[\lambda_{2} z_{1}\left(\lambda_{1}-\lambda_{3}\right)-\gamma_{2} z_{3}\left(\lambda_{2}-\lambda_{3}\right)+\lambda_{2} z_{2}\right] \\
& (*)_{2}=\lambda_{3} z_{1}^{2}\left(\lambda_{1}-\lambda_{3}\right)-\gamma_{3} z_{1} z_{3}\left(\lambda_{2}-\lambda_{3}\right) \\
& (*)_{3}=\lambda_{1} z_{1} z_{3}\left(\gamma_{3}-\gamma_{1}\right)-\gamma_{1} z_{3}\left[z_{2}-z_{3}\left(\gamma_{3}-\gamma_{2}\right)\right] \\
& (*)_{4}=\left(\gamma_{2} z_{3}-z_{2}\right)^{2}-\gamma_{2} \gamma_{3} z_{3}^{2} \\
& (*)_{5}=\gamma_{3} z_{3}\left[\left(\gamma_{2}-\gamma_{3}\right) z_{3}-z_{2}+\lambda_{3} z_{1}\right]-\lambda_{3} \gamma_{1} z_{1} z_{3}
\end{aligned}
$$

In this case the multiple model structures with $\lambda_{3}=\gamma_{3}=$ 0 should be avoided in order to ensure the observability condition (17) of the submodels.

Similar studies can be realized for the other possible cases, with one or two measures.

In the next section the observer design for the three-tank system is realized, taking into account the criterion choices of the multiple model presented in this section. 


\section{Observer design}

The method used here to design observers for nonlinear systems is based on the multiple model form (16). The case where the weighting functions $\mu_{i}$ depend on unmeasurable premise variables (state variables) is considered. Let us consider the matrices $A_{0}$ and $\overline{A_{i}}$ defined by:

$$
\begin{aligned}
& A_{0}=\frac{1}{r} \sum_{i=1}^{r} A_{i} \\
& \overline{A_{i}}=A_{i}-A_{0}
\end{aligned}
$$

By substituting $A_{0}$ and $\overline{A_{i}}$ in the multiple model state equation (16), the proposed observer for the three-tank system has the following form:

$$
\begin{aligned}
& \dot{\hat{x}}(t)=A_{0} \hat{x}(t)+\sum_{i=1}^{8} \mu_{i}(\hat{x})\left[\overline{A_{i}} \hat{x}(t)+B u(t)+L(y(t)-\hat{y}(t))\right] \\
& \hat{y}(t)=C \hat{x}(t)
\end{aligned}
$$

where $L$ is a matrix to be determined.

The state estimation error is given by:

$$
e(t)=x(t)-\hat{x}(t)
$$

Its dynamic is obtained using (21) and (20):

$$
\dot{e}(t)=\dot{x}(t)-\dot{\hat{x}}(t)=\left(A_{0}-L C\right) e(t)+\Delta(x, \hat{x})
$$

where:

$$
\begin{array}{r}
\Delta(x, \hat{x})=\sum_{i=1}^{r}\left[\overline{A_{i}}\left(\mu_{i}(x(t)) x(t)-\mu_{i}(\hat{x}(t)) \hat{x}(t)\right)\right. \\
\left.+B_{i}\left(\mu_{i}(x(t))-\mu_{i}(\hat{x}(t))\right) u(t)\right]
\end{array}
$$

can be considered as a small perturbation if $\hat{x}$ tends toward $x$. In conformity with [7] it is assumed that the following conditions hold:

$$
\begin{aligned}
& \left|\mu_{i}(x(t))-\mu_{i}(\hat{x}(t))\right|<\tau_{i}|x(t)-\hat{x}(t)| \\
& \left|\mu_{i}(x(t)) x(t)-\mu_{i}(\hat{x}(t)) \hat{x}(t)\right|<\omega_{i}|x(t)-\hat{x}(t)| \\
& |u(t)|<\varphi
\end{aligned}
$$

Since the weighting functions $\mu_{i}(x)$ take values in the interval $[0,1]$, are continuous and derivable, and the state $x$ is bounded, the conditions (24) are easy to check. The first condition in (24) is verified, since the weighting functions $\mu_{i}(x)$ are globally Lipschitz. Given a function $f$ of class $C^{1}$, we have:

$$
f(x)-f(\hat{x})=\int_{\hat{x}}^{x} \dot{f}(t) d t
$$

Applying this property for all eight weighting functions $\mu_{i}$ we obtain:

$$
\left|\mu_{i}(x)-\mu_{i}(\hat{x})\right| \leq \int_{\hat{x}}^{x}\left|\dot{\mu}_{i}(t)\right| d t \leq \tau_{i}|x-\hat{x}|, \quad i=1, \ldots, 8
$$

$\tau_{i}$ can be easily founded, knowing the extrema of functions $\mu_{i}(i=1, \ldots, 8)$. Since $\mu_{i}(x)$ are globally Lipschitz and $x$ is bounded and assumed to be continuous, the second condition in (24) is verified too. Applying the same principles for $f(x)=\mu_{i}(x) x$, the second one is checked similarly. In this case, the term $\Delta(x, \hat{x})$ may be bounded by:

$$
\Delta(x, \hat{x})<\psi|x(t)-\hat{x}(t)|
$$

where

$$
\psi=\sum_{i=1}^{r}\left(\bar{\sigma}\left(\overline{A_{i}}\right) \omega_{i}+\bar{\sigma}\left(B_{i}\right) \tau_{i} \varphi\right)
$$

where $\bar{\sigma}(M)$ denotes the maximum singular value of the matrix $M$. In conformity with the convergence theorem presented in [7] (Lemma 1), the state estimation error between the multiple model (4) and the multiple observer (20) converges asymptotically towards zero, if there exist matrices $P=P^{T}>0, Q=Q^{T}>0$ and $K$ such that the following condition holds:

$$
\left[\begin{array}{cc}
A_{0}^{T} P+P A_{0}-C^{T} K^{T}-K C+\psi^{2} Q & P \\
P & -Q
\end{array}\right]<0
$$

The gain of the observer (20) is then computed by:

$$
L=P^{-1} K
$$

It has to be remarked that the number of LMIs does not depend on the number of submodels of the multiple model. It depend only on $A_{0}$, the mean of these submodels $\left(A_{i}\right)$. Nevertheless, $A_{0}$ and $A_{i}$ have the same structure, so the choice of the multiple model is important in order to find $L$. The simulation results are given in figure 2 for $\lambda_{1}=-0.7$, $\lambda_{2}=5, \lambda_{3}=17, \gamma_{1}=0, \gamma_{2}=-3, \gamma_{3}=-1$ and $\psi=0.506$. These parameters have to be chosen in order to respect the observability conditions, as discussed previously. Considering different initial conditions for the multiple model $\left(x_{0}=[1.8 ; 1.7 ; 5.5]\right)$ and for the observer $\left(\hat{x}_{0}=[4.8 ; 5 ; 2.1]\right)$, a fast convergence of the state estimation error towards zero is obtained.
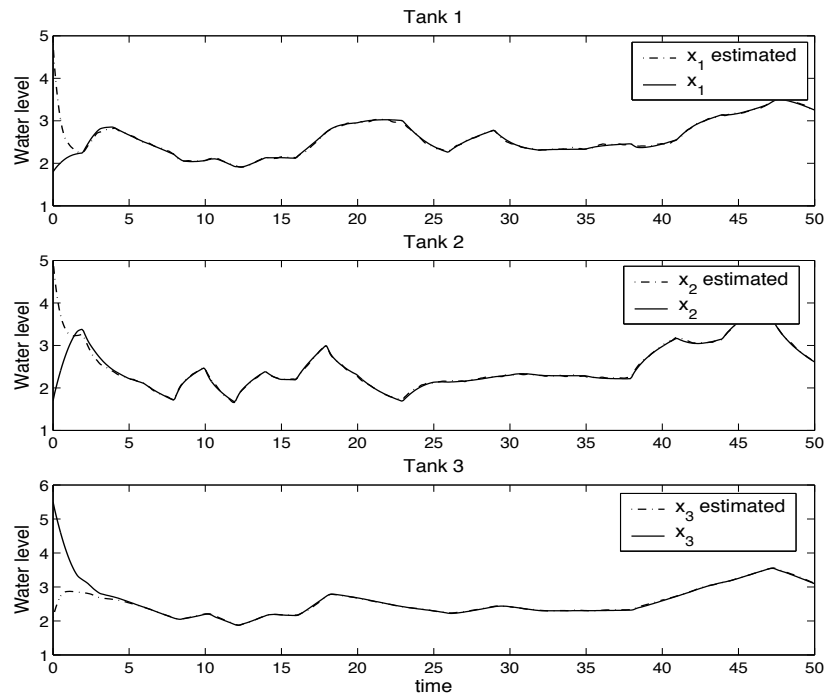

Fig. 2. State estimation for the three-tank system

The estimation results are correct (see figure 2), although a noise measurement has been added to the two outputs, as 
can be seen in figure 3. The LMI condition (28) holds and the observer gain is:

$$
L=\left[\begin{array}{cc}
1.42 & 0 \\
0 & 0.97 \\
-0.08 \cdot 10^{-2} & 0.12 \cdot 10^{-2}
\end{array}\right]
$$
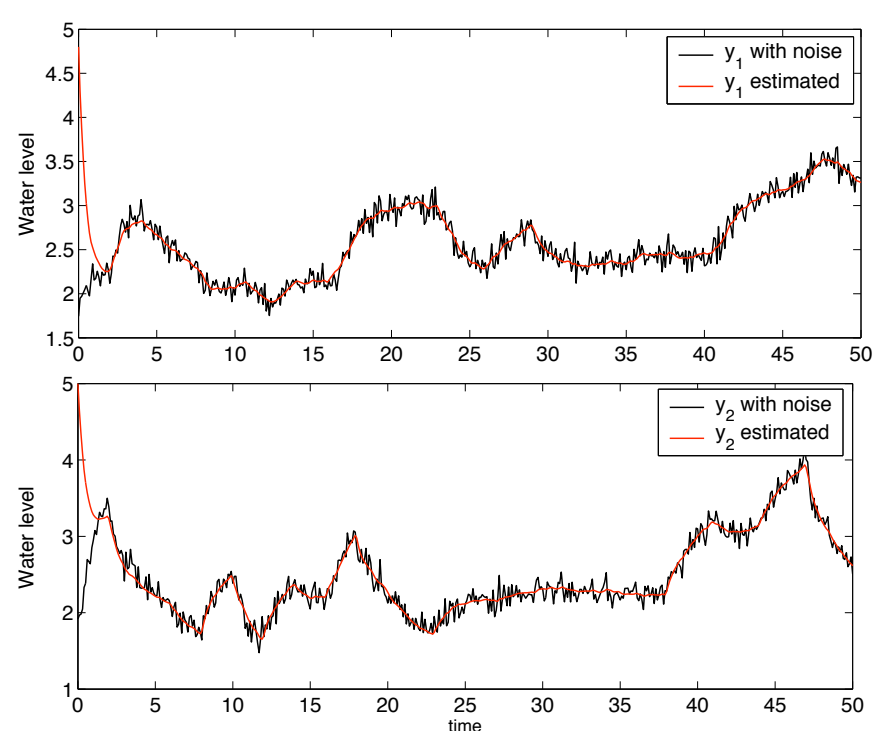

Fig. 3. Measured and reconstructed outputs

\section{CONCLUSIONS AND FUTURE WORKS}

This paper proposes an equivalent rewriting of nonlinear systems into a multiple model form with unmeasurable premise variables in order to design a multi-observer and reconstruct the state of this system. The transformation method is applied to the three-tank system and gives very good results concerning the state reconstruction.

The interest to use the multiple model structures is already well known, the main advantage is the ability to extend the tools of the linear theory to nonlinear systems. Other advantages and good properties of the multiple model are, firstly, the possibility to reduce the complexity of nonlinear systems, by constructing linear models that will be aggregated using weighting functions and, secondly, the fact that these aggregating functions possess the convexity property. The multiple model form developed here uses unmeasurable premise variables, the states of the system. In this way it covers a larger class of nonlinear systems than those using measurable premise variables and allows to develop only one model of the system behavior to detect and isolate actuator and sensor faults.

In order to obtain the multiple model structure, a method with no information loss is used. The initial nonlinear system is rewritten into an equivalent multiple model form. This method gives the possibility to determine between the different possible multiple models the one that is observable and for which the observer design is possible. These conditions are less restrictive than those proposed in the literature with regard to the Lipschitz constants. In addition, the number of LMIs to solve is restrained and does not depend on the number of submodels of the multiple model.

Using the method presented in this paper, it is envisaged to extend the study to fault detection and diagnosis.

\section{ACKNOWLEDGMENTS}

We acknowledge the financial support received from the "Fonds National de la Recherche Luxembourg". This research is also partially supported by the TASSILI no.07 program under MDU grant 714.

\section{REFERENCES}

[1] J. Abonyi, R. Babuska, F. Szeifert. Modified Gath-Geva fuzzy clustering for identification of Takagi-Sugeno fuzzy models. In IEEE Transactions on Systems, Man, and Cybernetics, Part B: Cybernetics, Vol. 32, No.5, 612-621, 2002.

[2] A. Akhenak, M. Chadli, J. Ragot, D. Maquin. Estimation of state and unknown inputs of a nonlinear system represented by a multiple model. In $11^{\text {th }}$ IFAC Symposium on Automation in Mining, Mineral and Metal Processing, MMM, Nancy, France, September 8-10, 2004.

[3] A. Akhenak, M. Chadli, J. Ragot, D. Maquin. Unknown input multiple observer based approach. Application to secure communications. In $1^{\text {th }}$ IFAC Conference on Analysis and Control of Chaotic Systems, Reims, France, June 28-30, 2006.

[4] P. Bergsten, R. Palm. Thau-Luenberger Observers for TS Fuzzy systems. In The Ninth IEEE International Conference on Fuzzy Systems, 2000.

[5] P. Bergsten, R. Palm, D. Driankov. Observers for Takagi-Sugeno Fuzzy Systems. In IEEE Transactions on Systems, Man and Cybernetics - Part B: Cybernetics, Vol 32, No 1, February 2002.

[6] M. Hou, Y. S. Xiong, R. J. Patton. Observing a Three-Tank System. In IEEE Transactions on Control Systems Technology, Vol. 13, No. 3, May 2005

[7] D. Ichalal, B. Marx, J. Ragot, D. Maquin. Design of Observers for Takagi-Sugeno Systems with Immeasurable Premise Variables: an L2 Approach. In $17^{\text {th }}$ World Congress The International Federation of Automatic Control, Seoul, Korea, July 6-11, 2008

[8] T. Johansen, R. Shorten, R. Murray-Smith. On the interpretation and identification of dynamic Takagi-Sugeno fuzzy models. In IEEE Transactions on Fuzzy Systems, Vol. 8, 297-313, 2000.

[9] A.M. Nagy, G. Mourot, J. Ragot, G. Schutz, S. Gillé. Model structure simplification of a biological reactor. In $15^{\text {th }}$ IFAC Symposium on System Identification, SYSID 2009, Saint-Malo, France, 6-8 July 2009.

[10] R. Palm, P. Bergsten. Sliding mode observers for Takagi-Sugeno Fuzzy Systems. In $9^{\text {th }}$ IEEE International Conference on Fuzzy Systems, FUZ'IEEE, 2000.

[11] J. Juan Rincon-Pasaye, R. Martinez-Guerra, A. Soria-Lopez. Fault diagnosis in nonlinear systems: An application to a three-tank system. In American Control Conference, Seattle, Washington, USA, June 1113,2008

[12] R.J. Patton, J. Chen, C.J. Lopez-Toribio. Fuzzy observers for nonlinear dynamic systems Fault diagnosis. In $37^{\text {th }}$ IEEE Conference on Decision and Control, Tampa, Florida USA, December 1998

[13] L. Rato, J.M. Lemos. Multimodel based fault tolerant control of the 3-Tank System. In European Control Conference, Invited Session, Karlsruhe, Germany, September 1999.

[14] K. Tanaka, H.O. Wang. Fuzzy Control System Design and analysis. A Linear Matrix Inequality Approach. In John Wiley \& Sons Inc., 2001

[15] K. Tanaka, H. Ohtake, H.O. Wang. A Descriptor System Approach to Fuzzy Control System Design via Fuzzy Lyapunov Functions. In IEEE Transactions on Fuzzy Systems, 15, 333-341, 2007.

[16] T. Takagi, M. Sugeno. Fuzzy identification of systems and its application to modeling and control. In IEEE Transactions on Systems, Man and Cybernet, Vol. 15, 166-172, 1985.

[17] D. Theilliol, H. Noura, J.-C. Ponsart. Fault diagnosis and accommodation of a three-tank system based on analytical redundancy. In ISA Transactions, No.41, 365-382, 2002. 